\title{
A DFT Study on the Structural and Electronic properties of Cadmium Telluride
} $\left(\mathrm{Cd}_{\mathrm{n}} \mathrm{Te}_{\mathrm{n}}\right)$ and Cadmium Zinc Telluride $\left(\mathrm{Cd}_{(\mathrm{n}-\mathrm{m})} \mathrm{Zn}_{\mathrm{m}} \mathrm{Te}_{\mathrm{n}}\right)$ Clusters

\author{
Menbere Weldetsadik $^{1}$ and Hagos Woldeghebriel ${ }^{2 *}$ \\ ${ }^{1}$ Department of Physics, CNCS, Wolkitie University, Wolkitie, Ethiopia, menbiaran@yahoo.com \\ ${ }^{2}$ Department of Physics, CNCS, P.O. Box 231, Mekelle University, Mekelle, Ethiopia \\ (*hagos93@mu.edu.et).
}

\begin{abstract}
In this study, the structural and electronic properties of $\mathrm{Cd}_{n} \mathrm{Te}_{\mathrm{n}}$ and $\mathrm{Cd}_{(\mathrm{n}-\mathrm{m})} \mathrm{Zn}_{\mathrm{m}} \mathrm{Te}_{\mathrm{n}}$ clusters have been studied using the plane wave based density functional theory (DFT). The QUANTUM ESPRESSO/PWSCF package employing the local density approximation (LDA) for the exchange correlation potential is used. In all calculations, the geometry optimization was employed in allowing the structures to fully relax. Structural properties viz. geometry, bond length and electronic properties like HOMO-LUMO gap, binding energy, second order energy difference and nature of bonding have been analyzed. As a result, we obtained that the binding energy increases with increasing cluster size and doping level. Zinc doped $\mathrm{Cd}_{\mathrm{n}} \mathrm{Te}_{\mathrm{n}}$ clusters show greater binding energy than the undoped clusters. Planar structures are obtained for very small cluster sizes and in our simulation three dimensional structure is found at $\mathrm{Cd}_{4} \mathrm{Te}_{4}$ cluster in the lowest energy geometry. Clusters of certain sizes often have special properties, i.e. higher stability or larger HOMO-LUMO gap when compared with other clusters, such as $\mathrm{Cd}_{(3-\mathrm{m})} \mathrm{Zn}_{\mathrm{m}} \mathrm{Te}_{3}$, $\mathrm{m}=0,1,2$ clusters. Thus, we can take it as a building block in the growth of the structures in our calculation. The partial charge density distribution of the HOMO and LUMO levels for $\mathrm{Cd}_{\mathrm{n}} \mathrm{Te}_{\mathrm{n}}$ and $\mathrm{Cd}_{(\mathrm{n}-\mathrm{m})} \mathrm{Zn}_{\mathrm{m}} \mathrm{Te}_{\mathrm{n}}$ clusters show that, the HOMO levels are predominantly localized on the Te atoms and the LUMO levels are distributed on both $\mathrm{Cd}$ and $\mathrm{Zn}$ atoms. Moreover, the LUMO levels are delocalized at the center of the clusters due to the hybridization of the molecular orbitals. The LDOS and energy level plots show discrete lines at the atomic level and the discreteness disappears as the cluster size increases.
\end{abstract}

Keywords: HOMO-LUMO, Pseudopotential, Cluster, Isomer.

\section{INTRODUCTION}

Semiconductor nanoparticles or Quantum Dots (QDs), in particular II-VI materials, have received tremendous attention during the last decades owing to their unusual physical properties and wide range of applications. Hence, a systematic study of QDs is useful to understand the evolution of their physical and chemical properties with size. The relationship between the geometry and the electronic structure plays a critical role in dictating the properties of a material. Semiconductor clusters have been shown to exhibit exotic properties quite different from those in molecules and solids. Compared with homogeneous clusters such as carbon and silicon, heterogeneous semiconductor clusters like CadmiumTelluride are more attractive because their properties can be controlled by changing the composition. For these reasons, theoretical studies 
on clusters are critical to the design and synthesis of advanced materials with desired optical, electronic and chemical properties.

The II-VI semiconductors are of great importance due to their applications in optoelectronics, solar cells, integrated optics and electro-optics devices. Hence, there are different experimental and theoretical studies on this group using various techniques or methods.

A number of theoretical and experimental attempts (Jianguang, 2009) have been made to determine the structure and properties of small $\mathrm{Cd}_{n} \mathrm{Te}_{\mathrm{n}}$ and related clusters. Most of the theoretical studies have been focused on clusters of a few atoms. According to Rusus (2006) on the study of structural, electronic transport and optical properties of Zn doped CdTe thin films, the non-doped CdTe thin films are found to be cubic oriented (111) polycrystalline structure whereas the doped once are a quasi-amorphous structure as investigated using X-Ray Diffraction techniques.

The structural, electronic and optical properties of CdTe clusters were studied using thermal evaporation and deposition method and Shimadzu UV-365 spectrophotometer in photon energy ranging from $0.5-2.5 \mathrm{eV}$. As a result, properties were dependent on the deposition parameter (Shreekanthan, 2006). Consequently, they mentioned low deposition rates were observed to result in hexagonal deposits whereas high rate of deposition favored cubic structure for the film. Similarly, Swati (2001) studied $\mathrm{Cd}_{(1-\mathrm{x})} \mathrm{Zn}_{\mathrm{x}} \mathrm{Te}$ crystals with $0 \leq \mathrm{x} \leq 0.14$ alloys using photoluminescence (PL) spectroscopy (PL) and found that, increasing the doping concentration increased the energy gap (Eg) of the bulk crystal. According to Bhattacharya and Anjali (2007), small stoichiometric $\mathrm{Cd}_{n} \mathrm{Te}_{\mathrm{n}}$ clusters and a few nonstoichiometric $\mathrm{Cd}_{\mathrm{m}} \mathrm{Te}_{\mathrm{n}}$ (for $\mathrm{m}, \mathrm{n}=1,4,13,16$, 19 and $\mathrm{m} \neq \mathrm{n}$ ) clusters have been studied using the density functional formalism and projector augmented wave method within the generalized gradient approximation. They observed that, upon relaxation, the symmetry changes for the Cd-rich clusters whereas the Te-rich clusters retain their symmetry.

Theoretical studies of heterogeneous semiconductor clusters have been limited due to computational difficulties arising from the large number of structural and permutational isomers formed due to multiple elements. On one hand, sophisticated computational methods such as self-consistent quantum mechanical calculation is required to make reliable prediction on the properties of these clusters in the absence of comprehensive experimental results. On the other 
hand, the amount of computational work is enormous in order to find all the stable isomers for a given cluster size and composition. Semiconductor nanoparticle materials are important in designing of novel photoelectrochemical devices based upon surface-derivetized nanoparticle films (Douglas et al., 1997).

In this work, we present a systematic study on the electronic and structural properties of Cadmium Telluride and Cadmium Zinc Telluride clusters using density functional theory.

\section{COMPUTATIONAL DETAILS}

First-principle calculations have been performed using pseudopotential method, as implemented in the QUANTUM ESPRESSO/ PWSCF package [http://www.pwscf.org], within density functional theory (DFT) (Hohenberg and Kohn, 1964; Kohn and Sham, 1965). Our calculations are based on a plane-wave expansion method employing the local density approximation (LDA) for exchange and correlation term approximated by the Ceperley and Alder (1980) functional and parameterized by Vosko et al. (1980). During structure optimization, we have used a cubic super cell of edge size $24 \AA$ and periodic boundary conditions are imposed. The simulation cell size is taken to be large in order to avoid interaction of ions from the adjacent cells. The plane wave cut-off was set to be $210 \mathrm{eV}$. In order to compare the results for various sizes, the Cadmium Telluride clusters are simulated with different number of Cadmium and Telluride paired atoms. We have employed conjugate gradient (CG) technique implemented for optimizing geometry. The valence electron configurations used for $\mathrm{Cd}, \mathrm{Zn}$ and Te are $5 \mathrm{~s}^{2} 4 \mathrm{~d}^{10}, 4 \mathrm{~s}^{2} 3 \mathrm{~d}^{10}$ and $5 \mathrm{~s}^{2} 5 \mathrm{p}^{4}$ respectively. In all the structure optimizations, the force and energy convergence considered are $10^{3} \mathrm{eV} / \mathrm{A}$ and $10^{4} \mathrm{eV}$ respectively.

\section{RESULTS AND DISCUSSION}

A major feature of semiconductor nanocrystals is the quantum confinement effect, which leads to spatial enclosure of the electronic charge carriers within the nanocrystals. Because of this effect, the size and shape of these artificial atoms widely and precisely tune the energy of discrete electronic energy states and optical transitions. These particles also span the transition between small molecules and bulk crystals. In the size regime of the nanocrystals where the size approaches the exciton (i.e. electron hole pairs) Bohr radius, the bulk semiconductor band 
structure with a continuum of energy states evolve into a set of discrete atomic-like energy states. Therefore, by simply changing the nanocrystal size, the optical properties of the material can be tuned (Swati, 2001). As the number of atoms in the particle increases, the discrete energies of the molecular orbitals merge toward a pseudo continuum of energy levels, converging to the solid state band-structure of the bulk material. The highest occupied molecular orbital minus the lowest unoccupied molecular orbital (HOMO-LUMO gap) energy increases with decreasing number of atoms in the cluster. This increasing energy difference as the size of the nanoparticle decreases is known as the quantum size effect. In this account, we discuss the structural and electronic properties of zinc doped and undoped cadmium telluride clusters, $\operatorname{Cd}_{\mathrm{n}} \operatorname{Te}_{\mathrm{n}}(n=1-7)$, and compare our calculations with other available similar results.

\subsection{Structural Properties of Cadmium Telluride $\left(\mathrm{Cd}_{n} \mathrm{Te}_{\mathrm{n}}\right)$ and Cadmium Zinc Telluride $\left(\mathbf{C d}_{(\mathrm{n}-\mathrm{m})} \mathrm{Zn}_{\mathrm{m}} \mathrm{Te}_{\mathrm{n}}\right)$ Clusters}

For the electronic structure calculations, we considered the binding energy, HOMO-LUMO energy gap and the second order energy difference explained below. The binding energy (BE) is the amount of energy released at its creation or the amount of energy we need to add to the system in order to break it up. So for, the cluster $\mathrm{Cd}_{\mathrm{n}} \mathrm{Te}_{\mathrm{n}}$, the binding energy per atom is calculated by using (Yonas and Hagos, 2014),

$$
B E=\frac{n[E(C d)+E(T e)]-E\left(C d_{n} T e_{n}\right)}{2 n}
$$

Where, $\mathrm{E}\left(\mathrm{Cd}_{\mathrm{n}} \mathrm{Te}_{\mathrm{n}}\right)$ is the lowest energy structure for $\mathrm{Cd}_{\mathrm{n}} \mathrm{Te}_{\mathrm{n}}$ cluster, $\mathrm{E}(\mathrm{Cd})$ and $\mathrm{E}(\mathrm{Te})$ are the energies of the cadmium and telluride atoms respectively and $n$ is the number of CdTe units.

This is used for hetrostructure, that is, made by two different atoms. For the $\mathrm{Zn}$ doped CdTe system, we use the following formula,

$$
B E=\frac{(n-m) E(C d)+n E(T e)+m E(Z n)-E\left(C d_{(n-m)}\right) Z n_{m} T e_{n}}{2 n}
$$

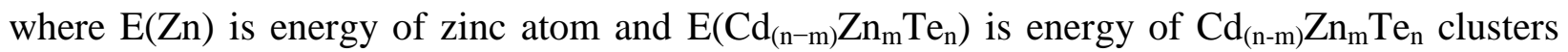
for $n=1-7$ and $m=0-3$.

There is another way of identifying the stability of clusters in cluster physics, that is, the secondorder energy difference. The second-order energy difference is a sensitive quantity that reflects 
the relative stability of cluster and can be directly compared with the experimental relative abundance and it can be calculated using (Hagos and Anjali, 2007),

$$
\Delta^{2} E=E_{(n-1)}+E_{(n+1)}-2 E_{(n)}
$$

where $\mathrm{E}_{(\mathrm{n}-1)}, \mathrm{E}_{(\mathrm{n}+1)}$ and $\mathrm{E}_{\mathrm{n}}$ represent the total energy of the lowest energy structure $\mathrm{Cd}_{(\mathrm{n}-1)} \operatorname{Te}_{(\mathrm{n}-1)}$, $\mathrm{Cd}_{(\mathrm{n}+1)} \mathrm{Te}_{(\mathrm{n}+1)}$ and $\mathrm{Cd}_{\mathrm{n}} \mathrm{Te}_{\mathrm{n}}$ clusters respectively.

The energy difference between the HOMO and LUMO reflects the ability for electrons to jump from occupied orbital to unoccupied orbital and represents the ability for the molecular orbital to participate in the chemical reactions to some extent. A larger HOMO-LUMO gap corresponds to a weaker chemical activity (Swati, 2001). This HOMO-LUMO gap energy is obtained by using equation (4):

$$
E g=(H O M O-L U M O)_{(\text {gap })}=E_{(L U M O)}-E_{(\text {HOMO })}
$$

Optimized geometry structures for $\mathrm{Cd}_{\mathrm{n}} \mathrm{Te}_{\mathrm{n}}$ for $n=1-7$ clusters are shown in figure 1 .

\subsubsection{CdTe dimer}

Cd-Te bond length of CdTe dimer is found to be $2.50 \AA$ which is in good agreement with the results obtained from GGA-PBE calculation found by Bhattacharya and Anjali (2007) $2.57 \AA$ and the binding energy calculated using equation (1) is $0.95 \mathrm{eV}$. The HOMO-LUMO gap energy calculated using equation (4) is $0.27 \mathrm{eV}$.

\subsection{2. $\mathrm{Cd}_{2} \mathrm{Te}_{2}$ cluster}

The lowest energy structure of this cluster attains a rhombus planar geometry. The Cd-Te bond length is found to be $2.69 \AA$ and that of $\mathrm{Cd}-\mathrm{Cd}$ is $2.73 \AA$. It favors ionic bonding due to the alternatively placed cadmium and telluride atoms in the cluster. Thus, the cluster has higher binding energy of $1.92 \mathrm{eV}$ per atom. This is due to the additional homonuclear Cd-Cd bonding. The HOMO-LUMO gap of this cluster is found to be $1.28 \mathrm{eV}$. It has two isomers that are first local minima in a square structure and second local minima in a rhombic planar structure with energy difference of $0.54 \mathrm{eV}$ and $1.03 \mathrm{eV}$ respectively; normalized to lowest energy structure.

\subsection{3. $\mathrm{Cd}_{3} \mathrm{Te}_{3}$ cluster}

This cluster presents alternate cadmium and telluride atoms, with three telluride atoms edge-

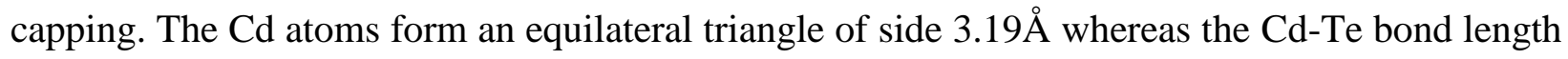
is found to be $2.62 \AA$. The binding energy per atom of this cluster is calculated to be $2.31 \mathrm{eV}$ and 
the HOMO-LUMO gap is $2.30 \mathrm{eV}$. From the above discussion, $\mathrm{Cd}_{3} \mathrm{Te}_{3}$ cluster has a greater binding and HOMO-LUMO energy gap.

\subsection{4. $\mathrm{Cd}_{4} \mathrm{Te}_{4}$}

The lowest energy structure of this cluster is a three dimensional (rhombohedral) structure made of two rhombus structure $\left(\mathrm{Cd}_{2} \mathrm{Te}_{2}\right)$, joined in a $\mathrm{Cd}$, Te alternating fashion. The average $\mathrm{Cd}-\mathrm{Te}$ bond length is calculated to be $2.80 \AA$ and this value is agreed with the bulk bond length $2.81 \AA$ (Bhattacharya and Anjali, 2007). The Cd-Te bond length increases as compared with that of the $\mathrm{Cd}_{3} \mathrm{Te}_{3}$ and this might be due to the increase in coordination number. The binding and HUMOLUMO gap energy of this cluster are found to be $2.37 \mathrm{eV}$ and $2.01 \mathrm{eV}$ respectively.

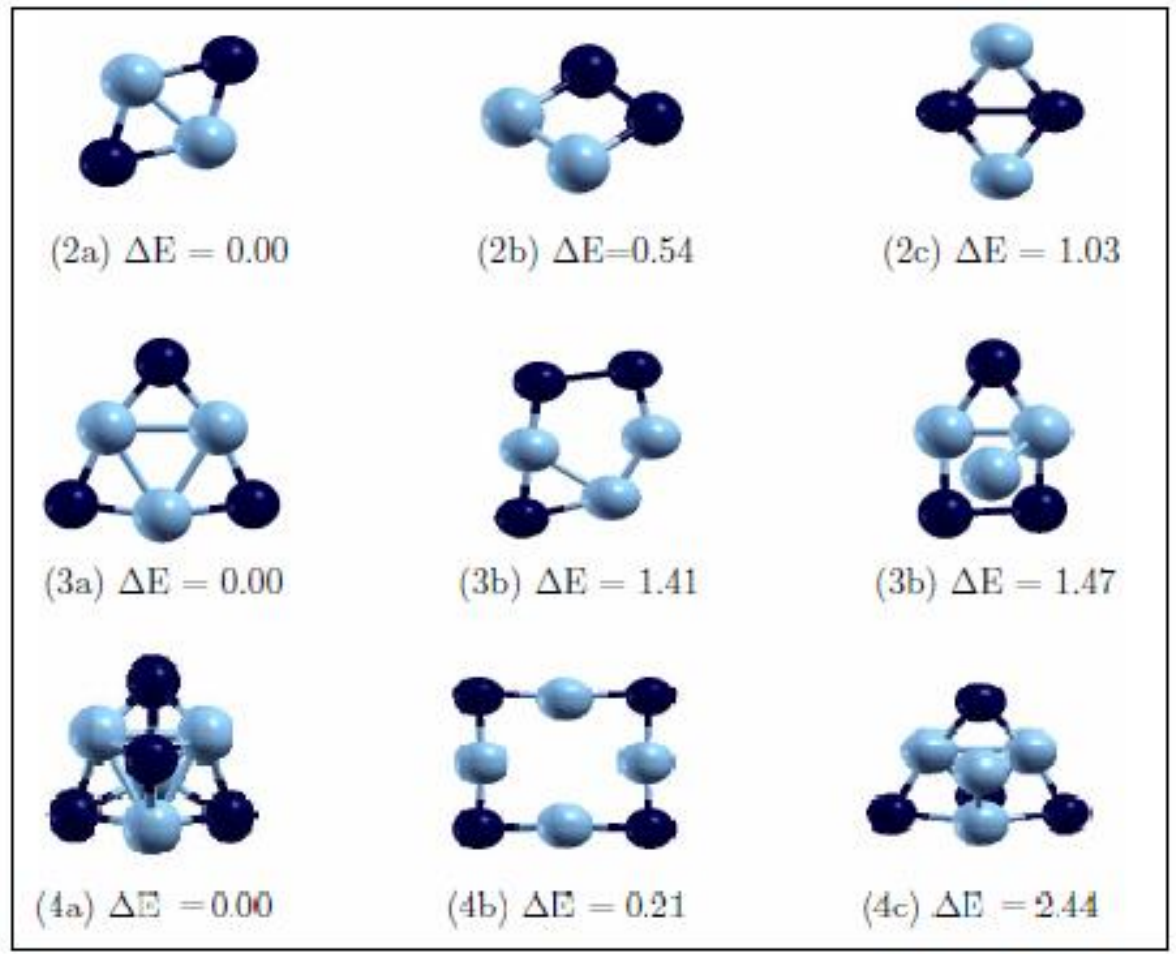

Figure 1. Optimized geometries for $\mathrm{Cd}_{n} \mathrm{Te}_{n}$ clusters for $\mathrm{n}=1-4$. (a) denote the lowest energy structures, (b) denote the next lowest energy structure(first isomer) and in (c) are the second isomers. Numbers at the bottom of each cluster represent total energy difference, in $\mathrm{eV}$, with respect to the lowest energy structure. (Dark color represents Te atom and the other is $\mathrm{Cd}$ atom).

From figure 1, we observe that, the isomers are found with the energy difference of $0.21 \mathrm{eV}$ and $2.44 \mathrm{eV}$ respectively with respect to the lowest energy configuration. The first isomeric structure is a square planar structure with $\mathrm{Cd}$ and $\mathrm{Te}$ atoms in an alternating arrangement, while the (C) CNCS, Mekelle University 
second isomeric structure is found by capping $\mathrm{Cd}$ and $\mathrm{Te}$ on the triangular structure of the lowest energy structure of $\mathrm{Cd}_{3} \mathrm{Te}_{3}$ cluster as illustrated in figure 1 .

\subsection{5. $\mathrm{Cd}_{5} \mathrm{Te}_{5}$}

The lowest energy structure is found from three rhombic and two triangular structures with an average CdTe bond length of $2.76 \AA$. It is derived from $\mathrm{Cd}_{4} \mathrm{Te}_{4}$ cluster by adding $\mathrm{CdTe}$ dimer along the negative $\mathrm{x}$-direction. The binding and the HOMO-LUMO energy gap of this cluster are calculated to be $2.44 \mathrm{eV}$ and $1.56 \mathrm{eV}$ respectively. As shown in figure 2, it has first and second lowest minimum energy structures with the energy difference of $1.18 \mathrm{eV}$ and $2.45 \mathrm{eV}$ respectively with respect to the lowest energy structure. The first lowest minimum energy structure is planar.

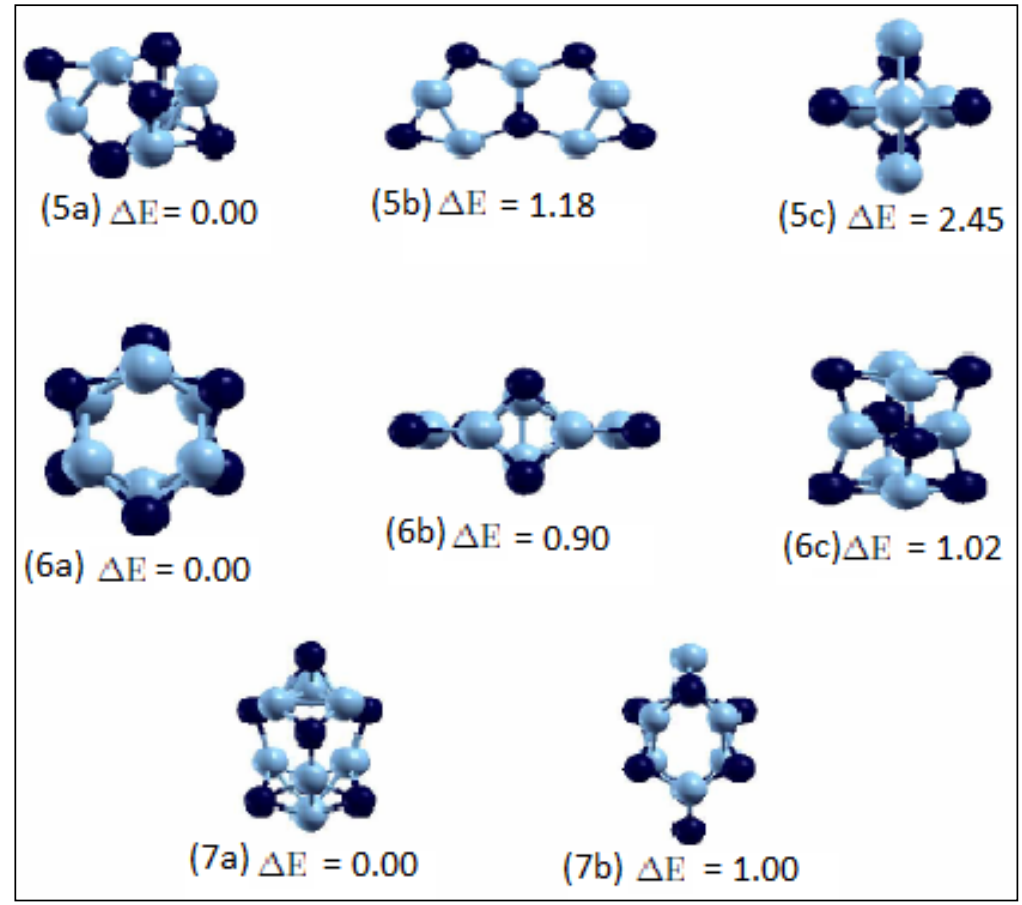

Figure 2. Optimized geometries for $\mathrm{Cd}_{n} \mathrm{Te}_{\mathrm{n}}$ clusters for $\mathrm{n}=5-7$. (a) denote the lowest energy structures, (b) denote the next lowest energy structure(first isomer) and in (c) are the second isomers. Numbers at the bottom of each cluster represent total energy difference, in $\mathrm{eV}$, with respect to the lowest energy structure. (Dark color denote Te atom and the other is $\mathrm{Cd}$ ).

\subsection{6. $\mathrm{Cd}_{6} \mathrm{Te}_{6}$}

The lowest energy structure is a hexagonal structure built from two parallel triangular structures joined together by six rhombus structures from a side. Its average $\mathrm{Cd}-\mathrm{Te}$ bond length is found to 
be $2.77 \AA$ with the binding and HOMO-LUMO energy gap of $2.54 \mathrm{eV}$ and $1.86 \mathrm{eV}$ respectively. Its first and second lowest minimum energy structures are both three dimensional.

\subsection{7. $\mathrm{Cd}_{7} \mathrm{Te}_{7}$ cluster}

This structure is obtained by capping $\mathrm{Cd}$ on one of the surfaces of $\mathrm{Cd}_{6} \mathrm{Te}_{6}$ polyhedron as shown in figure 2 (7a), Te on the other surface. As a result, six $\mathrm{Cd}_{2} \mathrm{Te}_{2}$ and three $\mathrm{Cd}_{3} \mathrm{Te}_{3}$ structures are

found in the lowest energy structure. The average Cd-Te distance is calculated to be $2.76 \AA$ and the binding energy per atom of this cluster is found to be $2.55 \mathrm{eV}$ with the HOMO-LUMO gap of $1.96 \mathrm{eV}$. We include one higher energy structure with an energy difference of $1.0 \mathrm{eV}$.

As shown in figures 1 and 2, the lowest energy structures are all three dimensional (except for $n$ $=2$ and 3) which are made up of rhombic and triangular structures. Moreover, we observe that, $\mathrm{Cd}_{4} \mathrm{Te}_{4}$ and $\mathrm{Cd}_{6} \mathrm{Te}_{6}$ clusters are more symmetric and compact than the other clusters. From the lowest energy structure of $\mathrm{Cd}_{\mathrm{n}} \mathrm{Te}_{\mathrm{n}}$ clusters discussed above and are shown in figures 1 and 2, we observe two basic building blocks in the construction of the structures, i.e. rhombic $\mathrm{Cd}_{2} \mathrm{Te}_{2}$ and triangular $\mathrm{Cd}_{3} \mathrm{Te}_{3}$ with $\mathrm{Cd}-\mathrm{Te}$ bond alternative arrangement. As the cluster size increases from $\mathrm{Cd}_{5} \mathrm{Te}_{5}$ to $\mathrm{Cd}_{7} \mathrm{Te}_{7}$, the number of triangular structure or $\mathrm{Cd}_{3} \mathrm{Te}_{3}$ clusters also increases. Therefore, the lowest-energy structure consisting of $\mathrm{Cd} 3 \mathrm{Te} 3$ with $\mathrm{CdTe}$ alternative arrangement can be viewed as the embryo of zinc-blende structure of cadmium telluride crystal. Moreover, because of the intensive direction-properties of the CdTe covalent bond, there is no radical change in the structures for $\mathrm{Cd}_{\mathrm{n}} \mathrm{Te}_{\mathrm{n}}$ clusters for $n=6$ and 7. This result agrees with the work of Jianguang (2009) except for $\mathrm{Cd}_{4} \mathrm{Te}_{4}$ and $\mathrm{Cd}_{5} \mathrm{Te}_{5}$ clusters in which they obtained planar structures as a lowest energy but in this study, the structure of these clusters are three dimensional as mentioned above. The difference might be the method they applied GGA-PBE. In general, the average CdTe bond length of the studied clusters is less than that of the bulk CdTe crystal, 2.81A. This might be due to the incomplete coordination in these clusters on account of very small number of atoms. The bond length of CdTe dimer is the smallest. The average Cd-Te bond length shrinks with increasing $n$ in the planar structures, $n=1-3$ and increases in going from planar to 3D structures.

\subsection{Lowest Energy Structures of $\left(\operatorname{Cd}_{(n-m)} Z_{m} T e_{n}\right)$, for $n=1-7$ and $m=0-3$ clusters}

Similar to the cadmium telluride bare clusters, we have performed energy optimization of cadmium zinc telluride clusters. The optimized structures of $\left(\mathrm{Cd}_{(\mathrm{n}-\mathrm{m})} \mathrm{Zn}_{\mathrm{m}} \mathrm{Te}_{\mathrm{n}}\right)$ clusters for $n=1-$ 
7 and $m=0-3$, with the condition that $n-m \geq m$ displayed in figure 3 are similar to the corresponding structures of bare $\mathrm{Cd}_{\mathrm{n}} \mathrm{Te}_{\mathrm{n}}$ clusters, discussed in the previous section. But we have found different values of $\mathrm{Zn}-\mathrm{Te}$ bond length after doping as compared with $\mathrm{Cd}-\mathrm{Te}$ bond length in the bare $\mathrm{Cd}_{\mathrm{n}} \mathrm{Te}_{\mathrm{n}}$ clusters.

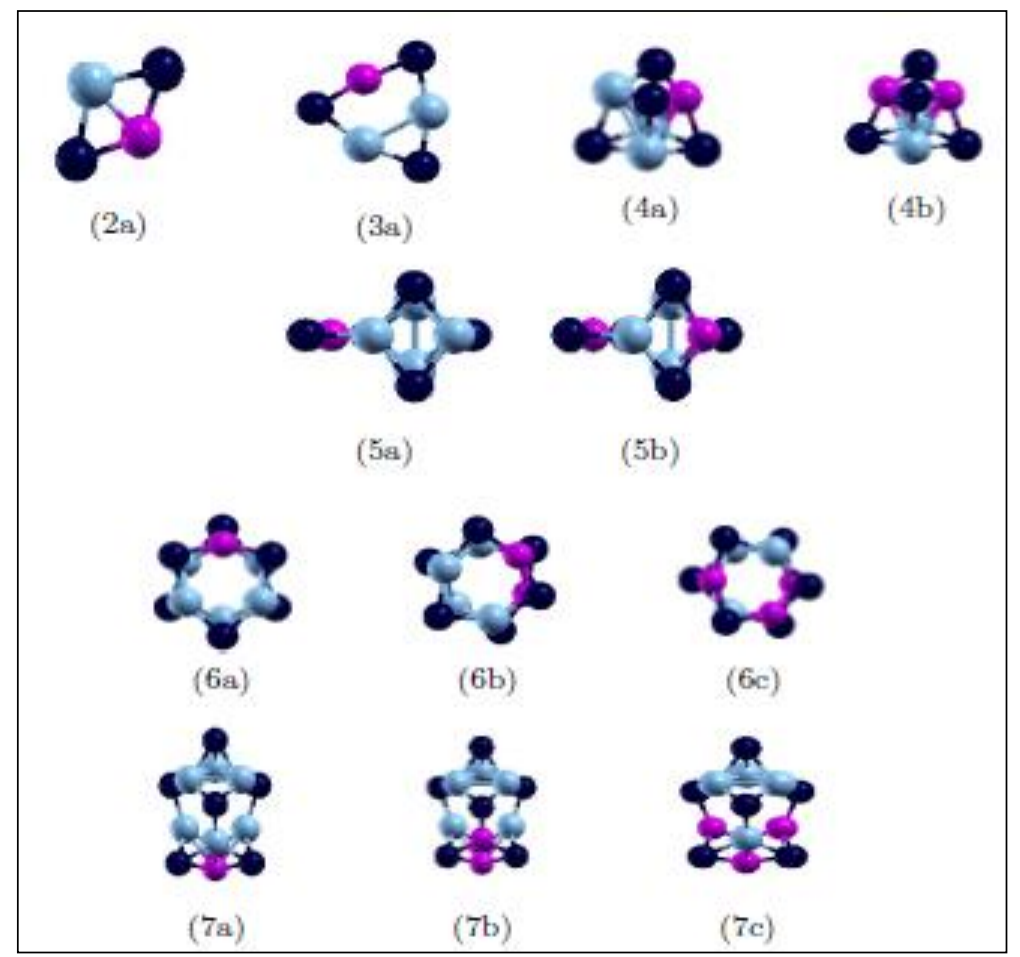

Figure 3. Optimized geometries of $\left(\mathrm{Cd}_{(\mathrm{n}-\mathrm{m})} \mathrm{Zn}_{\mathrm{m}} \mathrm{Te}_{\mathrm{n}}\right)$ for $\mathrm{n}=1-7$ and $\mathrm{m}=0-3$. (a) denotes optimized geometries of Monodoped $(\mathrm{m}=1)(\mathrm{b})$ denotes the optimized geometries of Bi-doped $(m=2)$ and $(c)$ denotes optimized geometries of tri-doped $(m=3)$. Numbers at the bottom indicate the cluster size (n). (Dark color represent Te, dark gray (online red) represents $\mathrm{Zn}$, and gray is for $\mathrm{Cd}$ atom.).

In the optimized structures of bare and $\mathrm{Zn}$ doped $\mathrm{Cd}_{\mathrm{n}} \mathrm{Te}_{\mathrm{n}}$ clusters, we have observed that, the $\mathrm{Te}$ atoms prefer to stay on the peripherals of the clusters while $\mathrm{Cd}$ atom prefer to go inside the cluster in relative sense. Comparing the preference of $\mathrm{Cd}$ and $\mathrm{Zn}$ atoms in $\mathrm{Cd}{ }_{(\mathrm{n}-\mathrm{m})} \mathrm{Zn}_{\mathrm{m}} \mathrm{Te}_{\mathrm{n}}$ clusters, it is found that, $\mathrm{Zn}$ shows more tendency to go inside the clusters as compared with $\mathrm{Cd}$ atoms. This may be due to the difference in atomic radius and electronegativity of the atoms. The atomic radius of $\mathrm{Cd}, \mathrm{Zn}$ and $\mathrm{Te}$ atoms are respectively, $1.71 \AA$, $1.53 \AA$ and $1.42 \AA$ and the electronegativity of $\mathrm{Cd}, \mathrm{Zn}$ and $\mathrm{Te}$ atoms are $1.52 \mathrm{eV}, 1.59 \mathrm{eV}$ and $2.16 \mathrm{eV}$ (Miessler and Tarr, 2003). These results are analyzed by observing the structures formed and by comparing the bond (C) CNCS, Mekelle University 
angles and bond length between the atoms. Thus, the angle at $\hat{T e}<\hat{C d}<\hat{Z n}$ is due to the difference in lone pair electrons. This implies that, $\mathrm{Zn}$ atom moves inward more than $\mathrm{Cd}$ atom and Te atom moves out ward compared with $\mathrm{Cd}$ and $\mathrm{Te}$ atoms.

\subsection{Electronic Properties of Cadmium telluride and Cadmium zinc telluride Clusters}

One of the main goals in cluster physics is to search for stable individual units that can serve as the elementary building blocks for electronic and optical nano devices. This stability can be verified in terms of energy such as binding energy, second order energy difference and HOMOLUMO gap energy. In this section, we have discussed the average $\mathrm{Cd}-\mathrm{Te}$ and $\mathrm{Zn}-\mathrm{Te}$ bond lengths, binding energy(BE), the HOMO-LUMO(HL) gap, second order energy differences (SOED), density of states (DOS) and partial charge density of cadmium telluride and cadmium zinc telluride clusters.

Table 1. The average Cd-Te bond length (in $\AA$ ) of $\mathrm{Cd}_{n} \mathrm{Te}_{\mathrm{n}}$ and $\mathrm{Cd}_{\mathrm{n}-\mathrm{m}} \mathrm{Zn}_{\mathrm{m}} \mathrm{Te}_{\mathrm{n}}$ clusters, for $\mathrm{n}=1$ 7 and $m=0-3$.

\begin{tabular}{|lllll|}
\hline Size, (n) & $\mathbf{L}(\mathbf{m}=\mathbf{0})$ & $\mathbf{L}(\mathbf{m}=\mathbf{1})$ & $\mathbf{L}(\mathbf{m}=\mathbf{2})$ & $\mathbf{L}(\mathbf{m}=\mathbf{3})$ \\
1 & 2.50 & & & \\
2 & 2.69 & 2.70 & & \\
3 & 2.62 & 2.62 & & \\
4 & 2.80 & 2.80 & 2.80 & \\
5 & 2.76 & 2.79 & 2.79 & \\
6 & 2.77 & 2.77 & 2.78 & 2.77 \\
7 & 2.76 & 3.77 & 3.77 & 2.77 \\
\hline
\end{tabular}

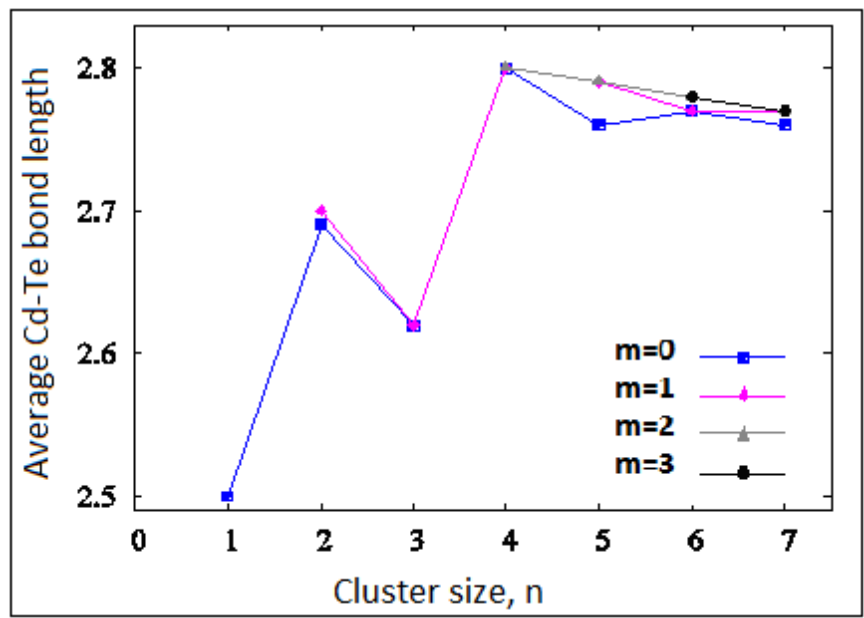

Figure 4. Graph of average $\mathrm{Cd}-\mathrm{Te}$ bond length (in ${ }^{\circ} \mathrm{A}$ ) of $\mathrm{Cd}_{\mathrm{n}} \mathrm{Te}_{\mathrm{n}}$ and $\mathrm{Cd}_{(\mathrm{n}-\mathrm{m})} \mathrm{Zn}_{\mathrm{m}} \mathrm{Te}_{\mathrm{n}}$ clusters, verses cluster size, $\mathrm{n}$ clusters, for $\mathrm{n}=1-7$ and $\mathrm{m}=0-3$. 
The variation of $\mathrm{Cd}-\mathrm{Te}$ bond length in the $\mathrm{Zn}$ doped clusters as compared with the undoped $\mathrm{Cd}_{\mathrm{n}} \mathrm{Te}_{\mathrm{n}}$ clusters is found to be insignificant except for $\mathrm{n}=5$ as shown in figure 4 . However, it is found in this work that, the $\mathrm{Zn}-\mathrm{Te}$ distance in the doped clusters is different from the $\mathrm{Cd}-\mathrm{Te}$ bond length due to the reasons given above, that is, ionic radius and electronegativity of the atoms.

Figure 4 shows the average bond length of $\mathrm{Cd}-\mathrm{Te}$ in doped and undoped $\mathrm{Cd}_{\mathrm{n}} \mathrm{Te}_{\mathrm{n}}$ clusters. From this figure, the average bond length in bare CdTe oscillates with increasing cluster size. The $\mathrm{Cd}$ Te bond length shows a dip at $\mathrm{Cd}_{3} \mathrm{Te}_{3}$ and $\mathrm{Cd}_{2} \mathrm{ZnTe}_{3}$ clusters. This might be due to the change in coordination number from two to three dimensional when compared with doped and undoped $\mathrm{Cd}_{4} \mathrm{Te}_{4}$ clusters. Thus, we can say that, these clusters might be stable clusters compared with the other clusters. The calculated values are shown in table 1 . If the cluster size increases in a planar structure, then, its bond length decreases. Most of the time, these planar structures make ionic bonds which have shorter bond lengths due to their strong force of attraction rather than covalent bonds. Our calculation shows that $\mathrm{CdTe}$ and $\mathrm{ZnTe}$ are most ionic clusters.

The binding energy of $\mathrm{Zn}$ doped and undoped $\mathrm{Cd}_{n} \mathrm{Te}_{\mathrm{n}}$ clusters are calculated using equation (1) and equation (2) and are given in table 2. Our calculation shows that, the binding energy of $\mathrm{Cd}_{\mathrm{n}} \mathrm{Te}_{\mathrm{n}}$ clusters increases with increasing cluster size and this may be due to the increase in coordination number. For the $\mathrm{Zn}$ doped clusters, the binding energy increases with increasing the level of dopant. This effect is as a consequence of the decrease of $\mathrm{Zn}-\mathrm{Te}$ bond length (dimer $2.32 \AA$ ) as compared with $\mathrm{Cd}-\mathrm{Te}$ bond length (dimer $2.50 \AA$ ), this means, zinc atom has smaller covalent radius than cadmium atom. Moreover, figure 5 shows that, the $\mathrm{Zn}$ doped $\mathrm{Cd}_{\mathrm{n}} \mathrm{Te}_{\mathrm{n}}$ clusters binding energy curve is enhanced for $n=7$ which can be considered as the most stable cluster. At $\mathrm{Cd}_{\mathrm{n}} \mathrm{Te}_{\mathrm{n}}$ the binding energy of the $\mathrm{Zn}$ doped clusters is slightly greater than the corresponding undoped clusters and increases with increasing the number of doped atoms.

As was discussed before, one way of identifying the stability of the clusters is second-order energy difference. The second-order energy difference of monodoped and undoped clusters are calculated using equation (3) and the results are displayed in table 3 and figure. 6. As a result, doped and undoped $\mathrm{Cd}_{3} \mathrm{Te}_{3}$ and $\mathrm{Cd}_{6} \mathrm{Te}_{6}$ clusters show peak values as compared with their neighboring clusters. Hence, we can say that, these clusters are more stable than the other clusters. 
Table 2. The binding energy per atom, in $\mathrm{eV}$, of $\mathrm{Cd}_{\mathrm{n}} \mathrm{Te}_{\mathrm{n}}$ and $\mathrm{Cd}(\mathrm{n}-\mathrm{m}) \mathrm{Zn}_{\mathrm{m}} \mathrm{Te}_{\mathrm{n}}$ clusters as a function of cluster size $n$, for $n=1-7$ and $m=0-3$.

\begin{tabular}{|ccccc|}
\hline Size, $(\mathbf{n})$ & $\mathbf{E}(\mathbf{m}=\mathbf{0})$ & $\mathbf{E}(\mathbf{m}=\mathbf{1})$ & $\mathbf{E}(\mathbf{m}=\mathbf{2})$ & $\mathbf{E}(\mathbf{m}=\mathbf{3})$ \\
1 & 0.95 & & & \\
2 & 1.92 & 2.02 & & \\
3 & 2.31 & 2.37 & & \\
4 & 2.37 & 2.42 & 2.47 & \\
5 & 2.44 & 2.48 & 2.52 & \\
6 & 2.54 & 2.57 & 2.60 & 2.63 \\
7 & 2.55 & 3.01 & 3.04 & 3.10 \\
\hline
\end{tabular}



Figure 5. Graph of binding energy of $\mathrm{Cd}_{n} \mathrm{Te}_{\mathrm{n}}$ and $\mathrm{Cd}_{(\mathrm{n}-\mathrm{m})} \mathrm{Zn}_{\mathrm{m}} \mathrm{Te}_{\mathrm{n}}$ clusters verses cluster size, $\mathrm{n}$ clusters, for $n=1-7$ and $m=0-3$.

Table 3. The second-order energy difference $\Delta^{2} E(m=0)$ for the undoped $\mathrm{Cd}_{\mathrm{n}} \mathrm{Te}_{\mathrm{n}}$ cluster and the monodoped, $\mathrm{Cd}_{(\mathrm{n}-1)} \mathrm{ZnTe}_{\mathrm{n}}$ clusters, for $\mathrm{n}=1-7$.

\begin{tabular}{|ccc|}
\hline Size, $(\mathbf{n})$ & $\boldsymbol{\Delta}_{\mathbf{2}} \mathbf{E}(\mathbf{m}=\mathbf{0})$ & $\boldsymbol{\Delta}_{\mathbf{2}} \mathbf{E}(\mathbf{m}=\mathbf{1})$ \\
2 & -0.39 & \\
3 & 1.01 & 0.97 \\
4 & -0.21 & -0.25 \\
5 & -0.72 & -0.64 \\
6 & 0.96 & 0.77 \\
7 & -0.74 & -0.60 \\
\hline
\end{tabular}




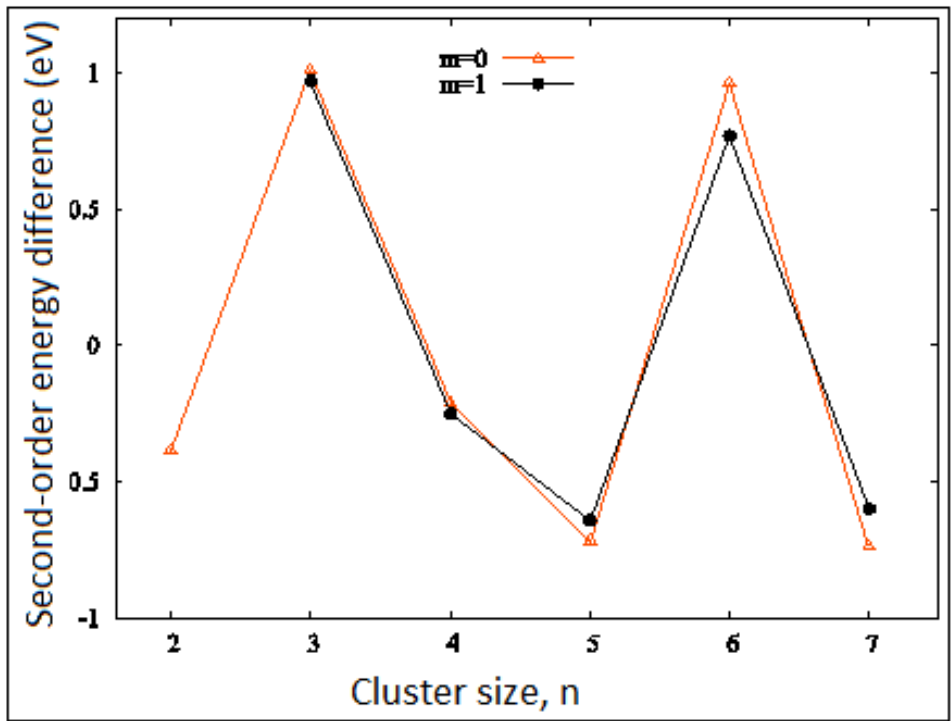

Figure 6. The second-order energy difference $m=0$ for the undoped $\mathrm{Cd}_{n} \mathrm{Te}_{\mathrm{n}}$ clusters and the monodoped, $\mathrm{Cd}_{(\mathrm{n}-1)} \mathrm{ZnTe}_{\mathrm{n}}$ clusters, for $\mathrm{n}=1-7$.

For the optimized configurations of doped and undoped $\mathrm{Cd}_{\mathrm{n}} \mathrm{Te}_{\mathrm{n}}$ clusters, the HOMO-LUMO energy gaps are computed and summarized in table 4. Clusters which have large HOMO-LUMO gap are more stable than those with lower values. The results shown in figure 7 are the HOMOLUMO gaps of those optimized cadmium telluride $\mathrm{Cd}_{\mathrm{n}} \mathrm{Te}_{\mathrm{n}}$ and cadmium zinc telluride clusters which do not show perfect odd/even oscillation. The HOMO-LUMO energy gap of bare $\mathrm{Cd}_{\mathrm{n}} \mathrm{Te}_{\mathrm{n}}$ clusters is included for comparison. From this figure, we can conclude that, as the cluster size increases, there is a tendency of increasing HOMO-LUMO gap which results in a less chemical reactivity. It is worthy to point out that, the HOMO-LUMO gap is also considered as an important parameter to estimate the electronic stability of small clusters. The larger energy gap indicates more electronic stability and less reactivity and vice versa. Therefore, in figure 7 the highest peak of HOMO-LUMO gaps appear at the $\mathrm{Cd}_{3} \mathrm{Te}_{3}$ and $\mathrm{Cd}_{2} \mathrm{ZnTe}_{3}$ clusters with the values of $2.30 \mathrm{eV}$ and $2.31 \mathrm{eV}$ respectively, implying that, these clusters possess the highest electronic stability than the other clusters. HUMO-LUMO gap of the doped and undoped $\mathrm{Cd}_{\mathrm{n}} \mathrm{Te}_{\mathrm{n}}$, for $n>2$ varies in the range of $1.26 \mathrm{eV}$ to $2.31 \mathrm{eV}$ which is in the range of the semiconductor band gap. The low HOMO-LUMO gaps appear at CdTe dimer. 
Table 4. HUMO-LUMO energy gap, (in eV), of $\mathrm{Cd}_{n} \mathrm{Te}_{\mathrm{n}}$ and $\mathrm{Cd}_{\mathrm{n}-\mathrm{m}} \mathrm{Zn}_{\mathrm{m}} \mathrm{Te}_{\mathrm{n}}$ clusters, for $\mathrm{n}=1-7$ and $\mathrm{m}=0-3$.

\begin{tabular}{|ccccc|}
\hline Size, (n) & $\mathbf{H}(\mathbf{m}=\mathbf{0})$ & $\mathbf{H}(\mathbf{m}=\mathbf{1})$ & $\mathbf{H}(\mathbf{m}=\mathbf{2})$ & $\mathbf{H}(\mathbf{m}=\mathbf{3})$ \\
1 & 0.27 & & & \\
2 & 1.28 & 1.26 & & \\
3 & 2.30 & 2.31 & & \\
4 & 2.01 & 1.90 & 1.88 & \\
5 & 1.56 & 1.62 & 1.62 & \\
6 & 1.86 & 1.83 & 1.84 & 1.81 \\
7 & 1.96 & 1.97 & 1.98 & 1.98 \\
\hline
\end{tabular}

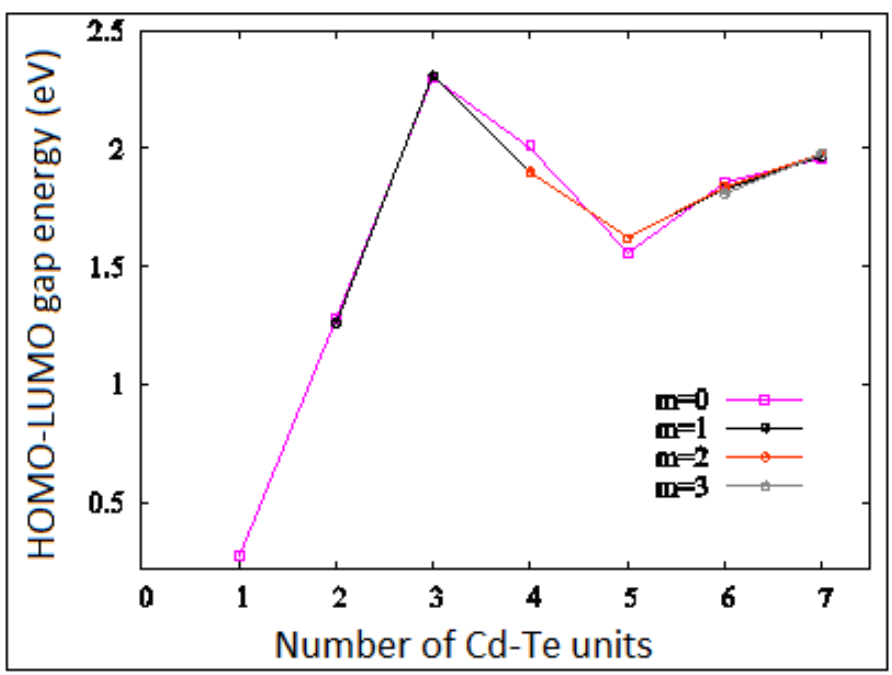

Figure 7. HOMO-LUMO energy gap (in eV) of $\mathrm{Cd}_{n} \mathrm{Te}_{\mathrm{n}}$ and $\mathrm{Cd}_{(\mathrm{n}-\mathrm{m})} \mathrm{Zn}_{\mathrm{m}} \mathrm{Te}_{\mathrm{n}}$ clusters, for $\mathrm{n}=1-$ 7 and $m=0-3$, as a function of cluster size (n).

\subsection{Partial Charge Density Analysis}

To describe the nature of bonding, delocalization of charge and other related properties, we have plotted isosurfaces of partial charge density plots of some representative clusters to bring out the contribution of each molecular orbital (MO) to the total charge density. It can be used to discuss the nature of individual molecular orbital, type of hybridization, overlap of molecular orbitals and delocalization of charge in the cluster and total charge density plots to understand the overall nature of bonding in the cluster.

\subsubsection{CdTe/ZnTe}

The bond length of CdTe and ZnTe dimers are found to be $2.50 \AA$ and $2.32 \AA$ which are smaller compared with the bulk values, with the binding energies of $0.95 \mathrm{eV}$ and $1.06 \mathrm{eV}$ respectively. 
The HOMO-LUMO gaps are calculated to be $0.27 \mathrm{eV}$ and $0.42 \mathrm{eV}$ respectively, where the Fermi levels are within the gap, slightly above the HOMO levels as shown in figure 8. These variations might be due to the properties of $\mathrm{Cd}$ and $\mathrm{Zn}$ atoms such as the ionic and covalent radii increase in going from $\mathrm{Zn}$ to $\mathrm{Cd}$ whereas electronegativity and ionization energies decrease. Since the radius of $\mathrm{Cd}$ is greater than that of $\mathrm{Zn}$, then the bond length of $\mathrm{Zn}-\mathrm{Te}$ is smaller than that of $\mathrm{Cd}-\mathrm{Te}$. Moreover, the binding energy and HOMO-LUMO energy gap of ZnTe is greater as compared with CdTe and this might be as a consequence of greater ionization energy and electronegativity of $\mathrm{Zn}$ atom. Partial charge density distributions of the HOMO and LUMO orbitals of the $\mathrm{CdTe}$ and $\mathrm{ZnTe}$ dimers are shown in figure 8.

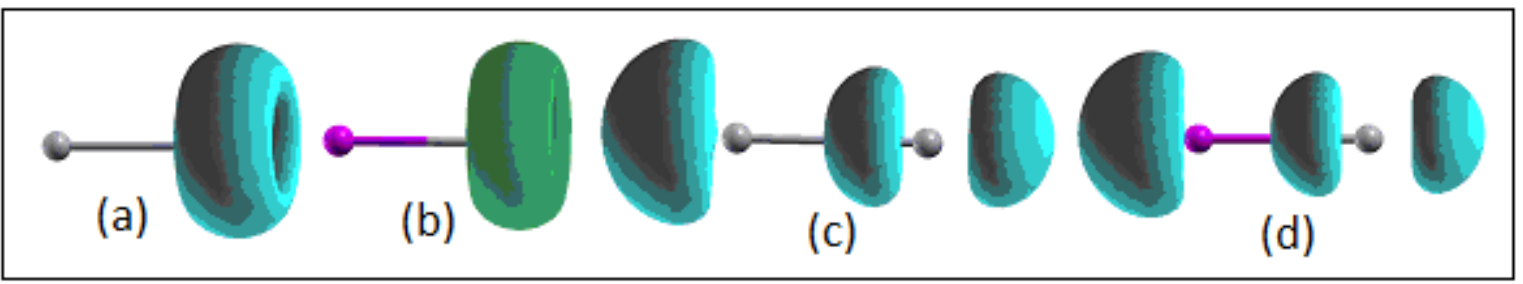

Figure 8. Partial charge density plots of the HOMO and LUMO orbitals of CdTe and ZnTe dimers. (a) and (b) are the HOMO of CdTe and ZnTe respectively and (c) and (d) show the LUMO of the two dimers respectively. Isosurfaces are plotted at $1 / 4^{\text {th }}$ of the maximum value.

Table 5. s, p and d partial charges with in $\mathrm{Cd}, \mathrm{Zn}$ and Te spheres calculated for some occupied and unoccupied orbitals in CdTe, (left), and ZnTe, (right) dimers.

\begin{tabular}{|c|c|c|c|c|c|c|c|c|c|}
\hline Orbital & Atom & $\mathbf{s}$ & p & d & Orbital & Atom & $\mathbf{s}$ & $\mathbf{p}$ & d \\
\hline \multirow[t]{2}{*}{ HOMO } & $\mathrm{Cd}$ & 0.00 & 0.02 & 0.01 & \multirow[t]{2}{*}{ HOMO } & $Z n$ & 0.00 & 0.02 & 0.01 \\
\hline & $\mathrm{Te}$ & 0.00 & 0.34 & 0.00 & & $T e$ & 0.00 & 0.30 & 0.00 \\
\hline \multirow[t]{2}{*}{ LUMO } & $\mathrm{Cd}$ & 0.08 & 0.10 & 0.01 & \multirow[t]{2}{*}{$L U M O$} & $Z n$ & 0.06 & 0.07 & 0.01 \\
\hline & $\mathrm{Te}$ & 0.00 & 0.19 & 0.00 & & $T e$ & 0.00 & 0.17 & 0.00 \\
\hline
\end{tabular}

From figure 8, we observe that, the HOMO of CdTe and ZnTe dimers are localized on the Te atom while the LUMOs are distributed on both the $\mathrm{Cd}$ and $\mathrm{Te}$ atoms with more contribution from the Te atoms. The HOMO consists of Te $p$ orbitals in both the dimers as shown in table 5. The LUMO of CdTe and ZnTe are found with the contribution of $s$ and $p$ orbitals of $\mathrm{Cd}$ and Te atoms to give $s p$-hybrid orbital which in turn forms a $\sigma$ bond with the Te $p$ orbital. 


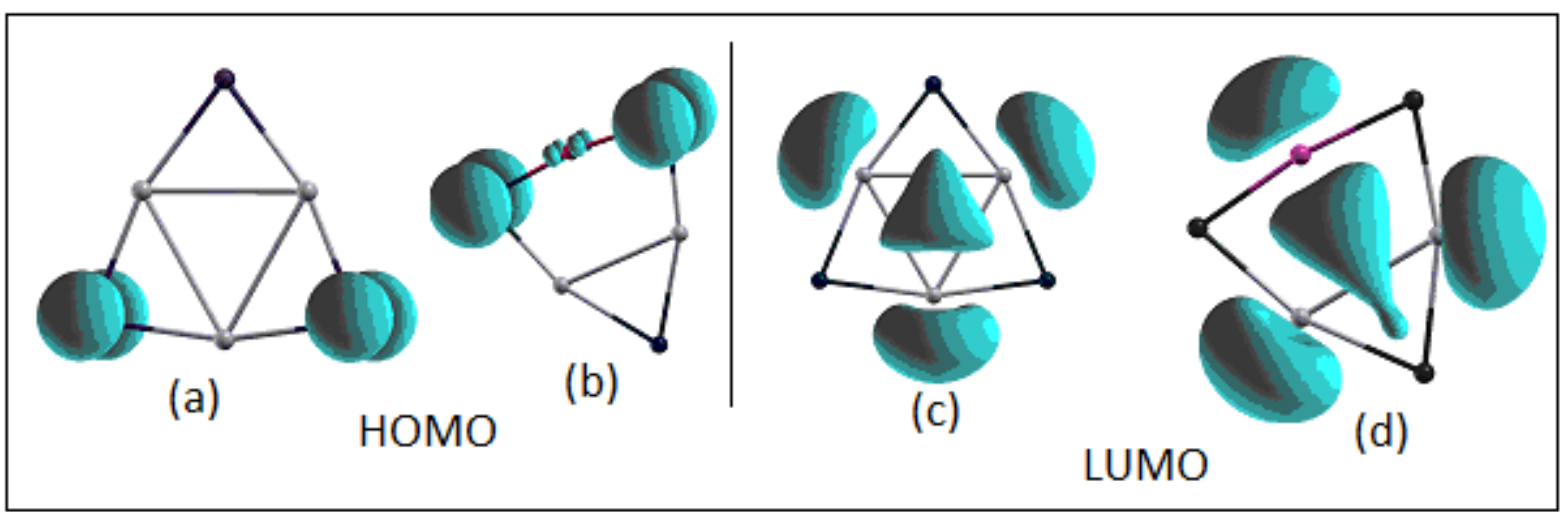

Figure 9. Partial charge density plots of orbitals around the Fermi level for $\mathrm{Zn}$ doped $\mathrm{Cd}_{3} \mathrm{Te}_{3}$ cluster and the bare $\mathrm{Cd}_{3} \mathrm{Te}_{3}$ for comparison. (a) and (c) are respectively HOMO and LUMO orbitals for $\mathrm{Cd}_{3} \mathrm{Te}_{3}$ and (b) and (d) are respectively LUMO orbitals for $\mathrm{Cd}_{2} \mathrm{ZnTe}_{3}$ clusters and Isosurfaces are plotted at $1 / 4^{\text {th }}$ of the maximum value. Cluster geometries are similar to figures $1(3 \mathrm{a})$ bare and 3 (3a) $\mathrm{Zn}$ doped.

Table 6. s, p and d partial charges with in $\mathrm{Cd}, \mathrm{Zn}$ and Te spheres calculated for some occupied and unoccupied orbitals in $\mathrm{Cd}_{3} \mathrm{Te}_{3}$ (a) and $\mathrm{Cd}_{2} \mathrm{ZnTe}_{3}$ (b).

\begin{tabular}{|c|c|c|c|c|c|c|c|c|c|}
\hline \multicolumn{5}{|c|}{ (a) } & \multicolumn{5}{|c|}{ (b) } \\
\hline Orbital & Atom & $\mathbf{S}$ & p & d & Orbital & Atom & $\mathbf{S}$ & p & d \\
\hline \multirow{6}{*}{ HOMO } & $\mathrm{Cd}$ & 0.00 & 0.01 & 0.00 & \multirow{6}{*}{ HOMO } & $\mathrm{Cd}$ & 0.00 & 0.01 & 0.00 \\
\hline & $\mathrm{Cd}$ & 0.00 & 0.01 & 0.00 & & $\mathrm{Cd}$ & 0.00 & 0.01 & 0.00 \\
\hline & $\mathrm{Cd}$ & 0.00 & 0.00 & 0.01 & & $\mathrm{Zn}$ & 0.00 & 0.00 & 0.02 \\
\hline & $\mathrm{Te}$ & 0.00 & 0.18 & 0.00 & & $\mathrm{Te}$ & 0.00 & 0.00 & 0.00 \\
\hline & $\mathrm{Te}$ & 0.00 & 0.18 & 0.00 & & $\mathrm{Te}$ & 0.00 & 0.16 & 0.00 \\
\hline & $\mathrm{Te}$ & 0.00 & 0.00 & 0.00 & & $\mathrm{Te}$ & 0.00 & 0.16 & 0.00 \\
\hline \multirow{6}{*}{ LUMO } & $\mathrm{Cd}$ & 0.02 & 0.06 & 0.00 & \multirow{6}{*}{ LUMO } & $\mathrm{Cd}$ & 0.02 & 0.08 & 0.00 \\
\hline & $\mathrm{Cd}$ & 0.02 & 0.06 & 0.00 & & $\mathrm{Cd}$ & 0.02 & 0.08 & 0.00 \\
\hline & $\mathrm{Cd}$ & 0.02 & 0.06 & 0.00 & & $\mathrm{Zn}$ & 0.01 & 0.06 & 0.00 \\
\hline & $\mathrm{Te}$ & 0.00 & 0.00 & 0.00 & & $\mathrm{Te}$ & 0.01 & 0.00 & 0.00 \\
\hline & $\mathrm{Te}$ & 0.00 & 0.00 & 0.00 & & $\mathrm{Te}$ & 0.00 & 0.00 & 0.00 \\
\hline & $\mathrm{Te}$ & 0.00 & 0.00 & 0.00 & & $\mathrm{Te}$ & 0.00 & 0.00 & 0.00 \\
\hline
\end{tabular}

\subsection{2. $\mathrm{Cd}_{3} \mathrm{Te}_{3} / \mathrm{Cd}_{2} \mathrm{ZnTe} \mathrm{T}_{3}$}

Figure 9 (a) and (b) show the $\mathrm{HOMO}$ of bare and $\mathrm{Zn}$ doped $\mathrm{Cd}_{3} \mathrm{Te}_{3}$ clusters respectively occupied at the two Te atoms, (c) and (d) show LUMO of bare and $\mathrm{Zn}$ doped $\mathrm{Cd}_{3} \mathrm{Te}_{3}$ clusters respectively occupied at the $\mathrm{Cd}$ atoms in the bare and $\mathrm{Cd}$ and $\mathrm{Zn}$ atoms in the doped cluster. It is found that, in these two clusters at the LUMO levels, charges are delocalized in the region between the atoms around the center. The detailed characteristics of partial charge distribution 
for these orbitals is displayed in table 6 which shows the atomic contribution to some of the energy levels around the Fermi level.

In figure 9, the partial charge density plot of the LUMO and LUMO+1 orbitals of both clusters (bare and doped) show delocalization of charges at the center of the clusters indicating their semi-metallic nature. Due to same number of valence electrons in $\mathrm{Cd}$ and $\mathrm{Zn}$ atoms, there a similarity between the HOMO and LUMO orbital distributions in the bare and $\mathrm{Zn}$ doped clusters as clearly shown in figure 9 and table 6 , which is also depicted in the dimers, figure 8 and table 5 .

\subsection{Density of States}

The density of states of electrons in bands of different clusters yield the number of states in a certain energy range. Energy level diagrams of $\mathrm{CdTe}, \mathrm{ZnTe}, \mathrm{Cd}_{3} \mathrm{Te}_{3}$ and $\mathrm{Cd}_{2} \mathrm{ZnTe}_{3}$ clusters are displayed in figure10 where the Fermi level is found between the HOMO and LUMO levels, very near to the HOMO levels.

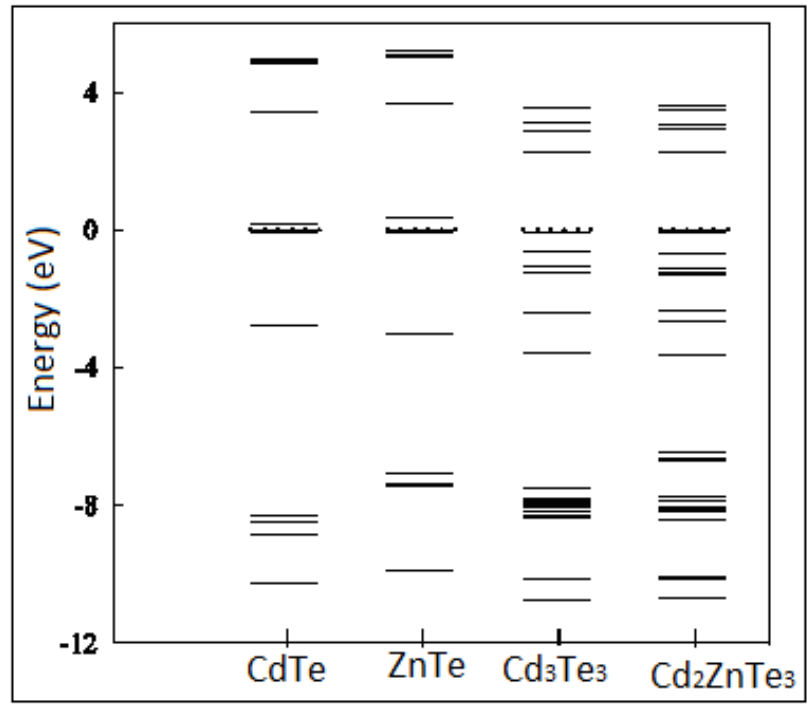

Figure 10. Energy levels of $\mathrm{Cd}_{3} \mathrm{Te}_{3}$ and $\mathrm{Cd}_{2} \mathrm{ZnTe}_{3}$ clusters. The discrete spectra are broadened by a Gaussian of width $0.01 \mathrm{eV}$.

As is shown clearly in figure 10, the LUMO levels of the doped clusters are shifted up ward with respect to the position of LUMO of bare clusters due to the properties of $\mathrm{Cd}$ and $\mathrm{Zn}$ atoms explained above. The high density of energy levels around $-8 \mathrm{eV}$ in $\mathrm{Cd}_{6} \mathrm{Te}_{6}$ cluster is observed to split into two groups with doping. As mention in Ali (2004) bond length and type of molecular orbitals are factors which affect the splitting of energy levels. The energy band gap of bulk CdTe 
crystal has a calculated value of $1.49 \mathrm{eV}$ (Rusu, 2006). From the energy level plots of selected clusters, it can be concluded that, the energy level spectrum of small size clusters (CdTe and $\mathrm{ZnTe}$ ) are more discrete than the large size clusters due to quantum confinement effect (Andrew and Shuming, 2010).

\section{CONCLUSION}

In this work structural and electronic properties of neutral and zinc doped cadmium telluride clusters are studied using QUANTUM ESPRESSO/ PWSCF package based on the principle of density functional theory (DFT), which in turn depends on pseudo-potential with a plane wave basis sets. The simulation of cadmium telluride and cadmium zinc telluride clusters is performed to study the size effect of the electronic and structural properties of the clusters. The lowestenergy structures of cadmium telluride clusters, $\mathrm{Cd}_{\mathrm{n}} \mathrm{Te}_{\mathrm{n}}$ for $\mathrm{n}=1-7$ have been considered. Binding energy, bond length, band gap (HOMO-LUMO) gap energy, the LDOS for some of the clusters, partial charge density of the clusters are discussed. Upon geometry optimization, cadmium zinc telluride clusters get slightly inward and outward relaxations, with $\mathrm{Cd}$ and $\mathrm{Zn}$ atoms pulled in and Te atoms pushed out. From the results we found that, the lowest energy structure is planar for $\mathrm{Cd}_{2} \mathrm{Te}_{2}$ and $\mathrm{Cd}_{3} \mathrm{Te}_{3}$ clusters while the remaining are three dimensional. Telluride atoms prefer to be positioned on the peripheral sides of the cluster, while cadmium and zinc atoms favor to go inside a cluster. Our calculation shows that, the binding energy increases with increasing cluster size, the fact that constituent atoms in larger clusters have more neighbors resulting in strong interactions, i.e. the surface effect decreases with cluster size. In our simulation, doping of clusters is found to increase the binding energy for the same number of $\mathrm{Te}$ atoms in the cluster. In addition, the bond length also changes with cluster size. The bond length of $\mathrm{Cd}$-Te dimer is greater than the bond length of $\mathrm{Zn}$-Te dimer. Clusters of certain sizes often have special properties, i.e. higher stability or larger HOMO-LUMO gap when compared with other clusters, such as $\mathrm{Cd}_{(3-\mathrm{m})} \mathrm{Zn}_{\mathrm{m}} \mathrm{Te}_{3}$, for $m=0,1,2$ clusters, according to the analysis of the binding energy, HOMO-LUMO gap and the second order energy difference. Thus, we can take it as a building block in the growth of the structures in our calculation. The partial charge density distribution of the HOMO and LUMO levels for $\mathrm{Cd}_{n} \mathrm{Te}_{\mathrm{n}}$ and $\mathrm{Cd}_{(\mathrm{n}-\mathrm{m})} \mathrm{Zn}_{\mathrm{m}} \mathrm{Te}_{\mathrm{n}}$ clusters show that the HOMO levels are predominantly localized on the Te atoms and the LUMO levels are 
distributed on both $\mathrm{Cd}$ and $\mathrm{Zn}$ atoms. Moreover, the LUMO levels are delocalized at the center of the clusters due to the hybridization of molecular orbitals. The LDOS and energy level plots show discrete levels change in nearly continuous spectra with increasing cluster size. We recommend at this point in time that a further work be done for large cluster size to see better characteristics of the clusters for practical applications using experiment and a high computing facility.

\section{ACKNOWLEDGEMNETS}

The authors would like to thank the Department of Physics at the College of Natural and Computational Sciences, Mekelle University, Ethiopia, for providing computational facilities to conduct the research work. The first author wants to thank the Ministry of Education of the Democratic Republic of Ethiopia for the financial support.

\section{REFERENCE}

Ali Omar, M. 2004. Elementary solid State Physics. Pearson Education, Inc., 176p.

Andrew, M. S \& Shuming, N. 2010. Semiconductor Nanocrystals: Structure, Properties and Band Gap Engineering. Acc. Chem. Res., 43(2): 190-200.

Bhattacharya, S.K \& Anjali, K. 2007. Ab-initio Calculations of Structural and Electronic Properties of CdTe Clusters. Phy. Rev., B 75: 035402.

Ceperley, D.M \& Alder, B. J. 1980. Ground State of the Electron Gas by a Stochastic Method. Phys. Rev. Lett., 45: 566.

Douglas L. S., Martin, P., Doug, H. R., Ed Urgiles., Andrew, F. C., David, W. N., Kim M. J., Randy J. E., Calvin J. C \& David S. G. 1997. CdTe Thin Films from Nanoparticle Precursors by Spray Deposition. Chem. Mater., 9: 889-900.

Hagos, W \& Anjali, K. 2007. How Cationic Gold Clusters respond to a single sulfur atom? J. Chem. Physics, 127(22): 224708.

Hohenberg, P \& Kohn, W. 1964. Inhomogeneous Electron Gas. Phys. Rev., 136: B864. http://www.pwscf.org, (PWSCF code in Quantum-Espresso).

Jianguang, W. 2009. Structural growth behavior and polarizability of CdTe clusters. Journal of Chemical Physics, 130: 214307. 
Kohn, W \& Sham, L. 1965. Self-Consistent Equations Including Exchange and Correlation Effects. Journal of Phys. Rev., 140: A1133.

Miessler, G.L \& Tarr, D. A. 2003. Inorganic Chemistry. $3^{\text {rd }}$ edition, Pearson Education, India $673 p$.

Rusus, G.G. 2006. Structural, Electronic Transport and Optical Properties of Zn-Doped CdTe Thin Films. Journal of Optoelectronics and Advanced Materials, 8: 931 - 935.

Shreekanthan, K. N. 2006. Structural and Properties of Vacuum Deposited Cadmium Telluride Thin Films, Indian Journal of Pure \& Applied Physics, 44: 706-708.

Swati, J. 2001. Photoluminescence Study of Cadmium Zinc Telluride. MSc Thesis, West Virginia University, Eberly College of Arts and Sciences (unpubl.).

Vosko, S.H., Wilk, L \& Nusair, M. C. 1980. Accurate spin-dependent electron liquid correlation energies for local spin density calculations: a critical analysis. J. Phys., 58:1200.

Yonas, M \& Hagos, W. 2014. Structural evolution and stabilities of $(\mathrm{PbTe})_{\mathrm{n}}(\mathrm{n}=1-20)$ clusters. Computational and Theoretical Chemistry, 1039: 40-49. 